\title{
Düsseldorfer Kreis
}

\section{Aufsichtsbehörde für den Datenschutz}

\section{Allgemeines}

Der Düsseldorfer Kreis stellt einen Zusammenschluss der gem $\$ 38$ BDSG zuständigen obersten Aufsichtsbehörden im nicht-öffentlichen Bereich dar. ${ }^{1}$ Durch diese Vereinigung soll die einheitliche Überwachung der Einhaltung des Datenschutzes durch alle Aufsichtsbehörden gewährleistet werden. Hierzu werden regelmäßig Treffen mit Vertretern der jeweiligen Aufsichtsbehörden abgehalten, bei denen aktuelle Probleme des Datenschutzrechts erörtert und Handlungshilfen formuliert werden. Die Aufsichtsbehörden der öffentlichen Bereiche gehören hingegen nicht zum Düsseldorfer Kreis, sondern bilden vielmehr in Form der Konferenz der Datenschutzbeauftragten des Bundes und der Länder eine eigene Organisation.

\section{Geschichte}

Bereits im Jahre 1976 wurde im Hinblick auf das geplante Bundesdatenschutzgesetz vom 1.2.1977, welches dann am 1.1.1979 in Kraft trat (BGBl. I, Nr. 7, 201), eine Arbeitsgemeinschaft der Vertreter der obersten Aufsichtsbehörden der Länder für den Datenschutz im nicht-öffentlichen Bereich gegründet. Deren Aufgabe sollte zunächst die einheitliche Auslegung und vor allem Anwendung des Bundesdatenschutzgesetzes sicherstellen. Im Herbst 1977 fand dann erstmalig das Treffen unter der Bezeichnung „Düsseldorfer Kreis“ statt. Grundlage für die Namenswahl war der damalige Tagungsort in Düsseldorf. Die folgenden Konferenzen fanden im Anschluss daran regelmäßig zwei Mal im Jahr unter dem Vorsitz des Innenministeriums Nordrhein-Westfalen (heute: Ministerium für Inneres und Kommunales des Landes Nordrhein-Westfalen) in Düsseldorf statt. Das änderte sich erstmalig mit der Konferenz am 1. Juli 2000 nach der Übernahme der Datenschutzaufsicht im nicht-öffentlichen Bereich durch das ULD in Schleswig-Holstein. Seitdem wechselt jährlich der Vorsitz und es wird nunmehr immer in den jeweiligen Teilnehmerländern getagt.

\section{Ziele und Aufgaben}

Hauptsächliches Ziel des Düsseldorfer Kreises ist der Informations- und Meinungsaustausch zu aktuellen Fragen des Datenschutzes sowie bei deren überregionaler Bedeutung die Erarbeitung einheitlicher Positionen. Hierzu werden bei den Treffen durch die Vertreter der Aufsichtsbehörden gegebenenfalls Rechtsauffassungen zu aktuellen Rechtsfragen des Datenschutzes dargelegt und sofern möglich Rechtsauslegungen aufeinander abgestimmt. Die Ergebnisse werden in Beschlüssen zusammengefasst, protokolliert und dann veröffentlicht. Die Beschlüsse der letzten Jahre können online eingesehen werden (vgl. bspw. auf den Seiten

1 Übersicht der Aufsichtsbehörden unter www.datenschutz.hessen.de/ adr_priv.htm\#entry1869 (letzter Abruf 9.3.2012). des Landesbeauftragten für Datenschutz und Informationsfreiheit von Nordrhein-Westfalen). ${ }^{2}$ Beschlüsse gibt es zum Beispiel zu den Themen „Datenschutz in sozialen Netzwerken“, „Beschäftigtenscreening bei AEO-Zertifizierung“, „Datenschutzgerechte Smartphone-Nutzung“ (2011), ,Schutz Minderjähriger in sozialen Netzwerken“ (2010), „Gesetzesänderung bei der Datenverwendung für Werbezwecke“ (2009) und „Versandhandel“ (2008). Gegenstand eines aktuellen Beschlusses des Düsseldorfer Kreises aus dem Jahre 2012 ist die „Einwilligungs- und Schweigepflichtentbindungserklärung in der Versicherungswirtschaft“.

\section{Arbeitsgemeinschaften}

Zur flächendeckenden Förderung des Datenschutzes in der Privatwirtschaft, sollen vor allem die Beziehungen zu großen Wirtschafts- und Dienstleistungsunternehmen ständig intensiviert und der Dialog über deren Dachverbände gefördert werden. Für einige Wirtschaftsbranchen wurden als direkte Ansprechpartner eigens spezielle Arbeitsgemeinschaften unter dem Vorsitz verschiedener Aufsichtsbehörden gebildet. Ständige Arbeitsgruppen gibt es beispielsweise für Auskunfteien, Internationalen Datenverkehr, Kreditwirtschaft, Technik, Telemedien und Versicherungswirtschaft. In diesen Arbeitsgruppen werden - über die regulären Treffen des Düsseldorfer Kreises hinaus - bereichsspezifische Probleme erörtert. Bei Bedarf können Vertreter der Privatwirtschaft zu einzelnen Themen eingeladen und gehört werden. Auf diese Weise wird versucht mit den jeweiligen Unternehmensverbänden Einvernehmen über datenschutzrechtliche Fragen zu erzielen. Über die vorgelegten Ergebnisse der Arbeitsgemeinschaften und deren Umsetzung entscheidet dann wiederum der Düsseldorfer Kreis in seinen Sitzungen.

\section{Bindungswirkung von Beschlüssen}

Die Beschlüsse des Düsseldorfer Kreises entfalten ungeachtet ihrer Protokollierung und Veröffentlichung keine unmittelbare Außenwirkung, d. h. keine zwingende rechtliche Bindungswirkung. Der Düsseldorfer Kreis stellt lediglich ein rein informelles Gremium dar und hat demzufolge auch nicht die Befugnis, durch Mehrheitsbeschluss zwingende Vorgaben für alle Aufsichtsbehörden durchzusetzen. Allerdings führen auf Unternehmensseite die Absprachen mit den Unternehmensvertretern zu einer freiwilligen Selbstbindung. Die Aufsichtsbehörden wiederum können einzelnen Beschlüssen des Düsseldorfer Kreises ausdrücklich zustimmen und sich auf diese Weise an die Absprachen binden. Eine solche Abstimmung besteht bspw. beim Genehmigungsverfahren zur Prüfung der Zulässigkeit von sogenannten Drittstaatentransfers nach $₫ 4$ c Abs. 2 BDSG.

2 Vgl.www.Idi.nrw.de/mainmenu_Service/submenu_Entschliessungsarchiv/ Inhalt/Beschluesse_Duesseldorfer_Kreis/index.php (letzter Abruf 9.3.2012). 